\title{
Doubly charged Higgsinos at LHC
}

\section{Durmuş A. DEMIR}

Department of Physics, Izmir Institute of Technology, IZTECH, TR35430 Izmir, Turkey

E-mail: demirephysics.iztech.edu.tr

\section{Mariana FRANK}

Department of Physics, Concordia University, 7141 Sherbrooke Street West, Montreal, Quebec, CANADA H4B 1 R6

E-mail: mfrankealcor.concordia.ca

\section{Katri HUITU*}

Department of Physics, University of Helsinki and Helsinki Institute of Physics, P.O. Box 64, FIN-00014 University of Helsinki, Finland

E-mail: katri.huitu@helsinki.fi

\section{Santosh Kumar RAI}

Department of Physics, University of Helsinki and Helsinki Institute of Physics, P.O. Box 64, FIN-00014 University of Helsinki, Finland

E-mail: santosh.rai@helsinki.fi

\section{Ismail TURAN}

Department of Physics, Concordia University, 7141 Sherbrooke Street West, Montreal, Quebec, CANADA H4B 1R6

E-mail: ituran@physics.concordia.ca

We review single and pair production and subsequent decay of doubly charged Higgsinos of the left-right supersymmetric model at the LHC. The novel signature is possible to separate from the MSSM and UED signal. 


\section{Introduction}

The Large Hadron Collider (LHC) will test ideas for beyond the Standard Model physics starting 2009. Triplet scalar fields occur in many extensions of the Standard Model (SM). Triplets come naturally in left-right symmetric models, based on the gauge group $S U(3)_{c} \times S U(2)_{L} \times S U(2)_{R} \times$ $U(1)$, where the triplet can provide the Majorana mass term for the neutrinos leading to the seesaw mass generation mechanism. Higgs sector in the left-right model needs similar fine tuning as in the Standard Model, and thus it is natural to supersymmetrize the model.

The triplets in supersymmetric models can contain doubly charged Higgsinos. These particles have several interesting properties. They do not mix with any other particle, as they are doubly charged fermions. Also, they do not have soft mass terms, and thus their mass is completely determined by a supersymmetric $\mu$ parameter. If this parameter is of the electroweak scale, the doubly charged Higgsino mass is also of the same scale, and can be one of the lightest supersymmetric particles [1]. Its signature in the collider experiment is relatively simple. In a part of the parameter space, it decays with almost $100 \%$ branching ratio to leptons and missing energy.

Other advantages of supersymmetric left-right (LRSUSY) models include automatic conservation of the $R$-parity, since $B-L$ is part of the gauge symmetry. Therefore, the lightest supersymmetric particle (LSP) is automatically stable and a candidate for dark matter [2]. The LSP is composed of neutral gauginos and Higgsinos, and it escapes the detector unnoticed. Therefore events with supersymmetric particles contain large amounts of missing energy. LRSUSY models provide also solution to the strong and weak $C P$ problems, which occur in the minimal supersymmetric model [3]. For more details of the model, see [4, 5].

In this talk, we discuss signals of doubly charged Higgsinos of a LRSUSY model at the LHC. We refer the reader to $[6,7]$ for a more detailed presentation of the analysis and model.

\section{The Left-Right Supersymmetric Model and Production of Doubly Charged Higgsinos}

The model is defined by the following superpotential

$$
\begin{aligned}
W= & \mathbf{Y}_{Q}^{(i)} Q^{T} \Phi_{i} i \tau_{2} Q^{c}+\mathbf{Y}_{L}^{(i)} L^{T} \Phi_{i} i \tau_{2} L^{c}+i\left(\mathbf{h}_{l l} L^{T} \tau_{2} \delta_{L} L+\mathbf{h}_{l l} L^{c T} \tau_{2} \Delta_{R} L^{c}\right) \\
& +\mu_{3} \operatorname{Tr}\left[\Delta_{L} \delta_{L}+\Delta_{R} \delta_{R}\right]+\mu_{i j} \operatorname{Tr}\left[i \tau_{2} \Phi_{i}^{T} i \tau_{2} \Phi_{j}\right]+W_{N R},
\end{aligned}
$$

where $W_{N R}$ denotes (possible) non-renormalizable terms arising from integrating-out of the heavier fields. The Higgs triplets are given by

$$
\Delta_{L}=\left(\begin{array}{cc}
\frac{1}{\sqrt{2}} \Delta_{L}^{-} & \Delta_{L}^{0} \\
\Delta_{L}^{--} & -\frac{1}{\sqrt{2}} \Delta_{L}^{-}
\end{array}\right) \sim(1,3,1,-2), \delta_{L}=\left(\begin{array}{cc}
\frac{1}{\sqrt{2}} \delta_{L}^{+} & \delta_{L}^{++} \\
\delta_{L}^{0} & -\frac{1}{\sqrt{2}} \delta_{L}^{+}
\end{array}\right) \sim(1,3,1,2),
$$

and similar multiplets for the the right-handed triplets.

The doubly charged Higgsinos can be produced in pairs at the LHC in the process $p p \longrightarrow \widetilde{\Delta}^{++} \widetilde{\Delta}^{--}$ via $s$-channel $\gamma$ and $Z_{L, R}$ exchanges, and singly in the process $p p \longrightarrow \tilde{\chi}_{1}^{+} \widetilde{\Delta}^{--}$via $s$-channel $W_{L, R}$ 


\begin{tabular}{|c|c|c|c|}
\hline Fields & $\begin{array}{c}\text { SPA } \\
\tan \beta=5, M_{B-L}=25 \mathrm{GeV} \\
M_{L}=M_{R}=250 \mathrm{GeV} \\
v_{\Delta_{R}}=3000 \mathrm{GeV}, \mathrm{v}_{\delta_{\mathrm{R}}}=1000 \mathrm{GeV} \\
\mu_{1}=1000 \mathrm{GeV}, \mu_{3}=300 \mathrm{GeV}\end{array}$ & $\begin{array}{c}\text { SPB } \\
\tan \beta=5, M_{B-L}=100 \mathrm{GeV} \\
M_{L}=M_{R}=500 \mathrm{GeV} \\
v_{\Delta_{R}}=2500 \mathrm{GeV}, \mathrm{v}_{\delta_{\mathrm{R}}}=1500 \mathrm{GeV} \\
\mu_{1}=500 \mathrm{GeV}, \mu_{3}=500 \mathrm{GeV}\end{array}$ & $\begin{array}{c}\text { SPC } \\
\tan \beta=5, M_{B-L}=0 \mathrm{GeV} \\
M_{L}=M_{R}=500 \mathrm{GeV} \\
v_{\Delta_{R}}=2500 \mathrm{GeV}, \mathrm{v}_{\delta_{\mathrm{R}}}=1500 \mathrm{GeV} \\
\mu_{1}=500 \mathrm{GeV}, \mu_{3}=300 \mathrm{GeV}\end{array}$ \\
\hline \multirow{4}{*}{$\begin{array}{c}{\widetilde{\chi_{i}^{0}}}_{0}^{0}(i=1,3) \\
\widetilde{\chi}_{i}^{ \pm}(i=1,3) \\
M_{\tilde{\Delta}} \\
W_{R}, Z_{R}\end{array}$} & $89.9,180.6,250.9 \mathrm{GeV}$ & $212.9,441.2,458.5 \mathrm{GeV}$ & $\overline{142.5,265.6,300.0 \mathrm{GeV}}$ \\
\hline & $250.9,300.0,953.9 \mathrm{GeV}$ & $459.4,500.0,500.0 \mathrm{GeV}$ & $300.0,459.3,500.0 \mathrm{GeV}$ \\
\hline & $300 \mathrm{GeV}$ & $500 \mathrm{GeV}$ & $300 \mathrm{GeV}$ \\
\hline & $2090.4,3508.5 \mathrm{GeV}$ & $1927.2,3234.8 \mathrm{GeV}$ & $1927.2,3234.8 \mathrm{GeV}$ \\
\hline & S2 & S3 & S3 \\
\hline$\widetilde{e}_{L}, \widetilde{e}_{R}$ & $(156.9 .155 .6 \mathrm{GeV}),(402.402 \mathrm{GeV})$ & $(254.2,253.4 \mathrm{GeV}),(552,552 \mathrm{GeV})$ & $(214.9,214.0 \mathrm{GeV}),(402.6,402.2 \mathrm{GeV})$ \\
\hline$\tilde{\mu}_{L}, \tilde{\mu}_{R}$ & $(156.9,155.6 \mathrm{GeV}),(402.402 \mathrm{GeV})$ & $(254.2,253.4 \mathrm{GeV}),(552,552 \mathrm{GeV})$ & $(214.9,214.0 \mathrm{GeV}),(402.6,402.2 \mathrm{GeV})$ \\
\hline$\tilde{\tau}_{1}, \widetilde{\tau}_{2}$ & $(155.4,159.9 \mathrm{GeV}),(401.406 \mathrm{GeV})$ & $(252.5,257.9 \mathrm{GeV}),(550,556 \mathrm{GeV})$ & $(212.8,216.2 \mathrm{GeV}),(401.5,403.3 \mathrm{GeV})$ \\
\hline
\end{tabular}

Table 1: The numerical values assigned to the model parameters in defining the sample points SPA, SPB and SPC. In each case, $\mathbf{S 2}$ and $\mathbf{S 3}$ designate parameter values which allow for 2-body and 3-body decays of doubly-charged Higgsinos, respectively. The VEVs of the left-handed Higgs triplets are taken as $v_{\Delta_{L}} \sim$ $v_{\delta_{L}} \simeq 10^{-8} \mathrm{GeV}$. For the couplings we use $g_{L}=g_{R}=g$ and for $h_{l l}=0.1$ [7].
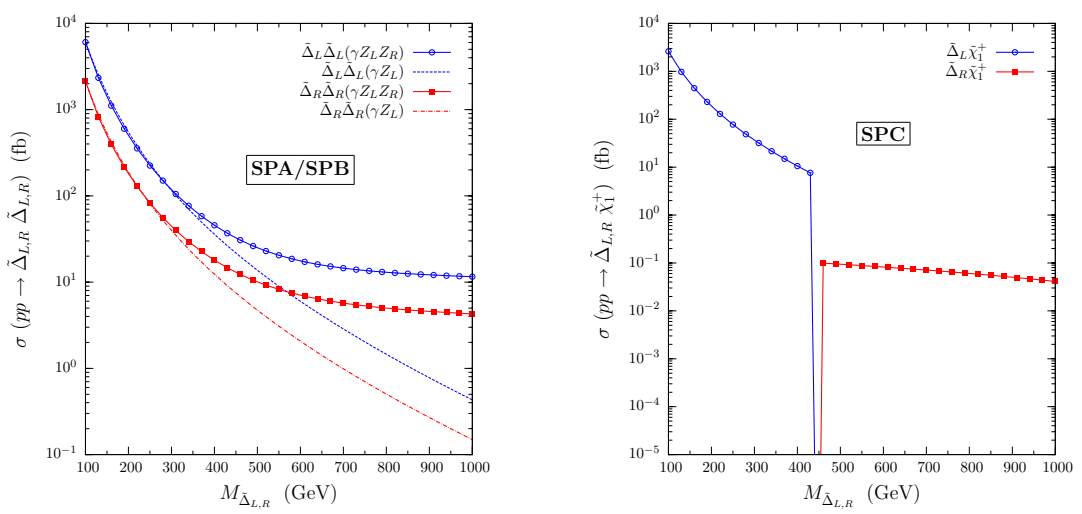

Figure 1: Left: The pair production cross sections for doubly charged Higgsinos at the LHC. Right: The single production cross sections of doubly charged Higgsinos at the LHC.

exchanges. These doubly and singly charged fermions subsequently decay via a chain of cascades until the lightest neutralino $\chi_{1}^{0}$ is reached. In general, the two-body decays of doubly charged Higgsinos are given by $\widetilde{\Delta}^{--} \longrightarrow \widetilde{\ell}^{-} \ell^{-}, \Delta^{--} \tilde{\chi}_{i}^{0}, \widetilde{\chi}_{i}^{-} \Delta^{-}, \tilde{\chi}_{i}^{-} W^{-}$, and these decay products further cascade into lower-mass daughter particles of which leptons are of particular interest. We consider regions of parameter space where the Higgs bosons are heavier than the doubly charged Higgsinos and find the decay width to $W^{-}$much smaller than the one to leptons.

We choose three sample points in the LRSUSY parameter space, as tabulated in Table 1. The sparticle mass spectrum suggests that the chargino states are also heavier than or comparable to the doubly charged Higgsinos. For the benchmark points in Table 1, the doubly charged Higgsinos have the following 2- $(\mathbf{S 2})$ and 3-body $(\mathbf{S 3})$ decay branching ratios $(i=e, \mu, \tau)$ :

$$
\begin{aligned}
& B R\left(\widetilde{\Delta}_{L / R}^{--} \rightarrow \tilde{\ell}_{i L / i R}^{-} \ell_{i}^{-}\right) \simeq \frac{1}{3}, B R\left(\tilde{\ell}_{i L / i R}^{-} \rightarrow \ell_{i}^{-} \widetilde{\chi}_{1}^{0}\right)=1, \quad m_{\tilde{l}_{i}}<M_{\tilde{\Delta}^{--}} \\
& B R\left(\widetilde{\Delta}_{L / R}^{--} \rightarrow \ell_{i}^{-} \ell_{i}^{-} \widetilde{\chi}_{1}^{0}\right) \simeq \frac{1}{3}, \quad m_{\tilde{l}_{i}}>M_{\tilde{\Delta}^{--}} .
\end{aligned}
$$

The pair-production and associated production cross sections are shown in Fig 1. Assuming only 

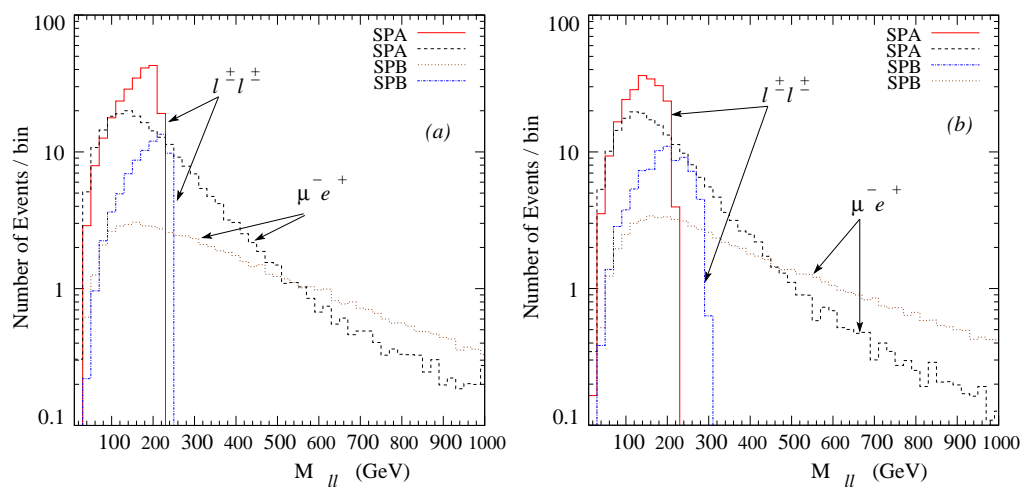

Figure 2: Binwise invariant mass distribution of lepton pairs with integrated luminosity of $\int \mathscr{L} d t=30 \mathrm{fb}^{-1}$. The panel (a) represents the 2-body (S2) case, and panel (b) the 3-body (S3) case.

diagonal couplings $h_{l l}$, the doubly charged Higgsinos decay into two same-sign same-flavor (SSSF) leptons and the lightest neutralino $\widetilde{\chi}_{1}^{0}$, the LSP. This decay pattern, when the doubly charged states are produced in pairs, gives rise to final states involving four isolated leptons of the form

$$
p p \longrightarrow \widetilde{\Delta}^{++} \widetilde{\Delta}^{--} \longrightarrow\left(\ell_{i}^{+} \ell_{i}^{+}\right)+\left(\ell_{j}^{-} \ell_{j}^{-}\right)+H_{T},
$$

where $\ell_{i}, \ell_{j}=e, \mu, \tau$. Similarly, in the case of associated production one gets

$$
p p \longrightarrow \widetilde{\Delta}^{--} \tilde{\chi}_{1}^{+} \longrightarrow\left(\ell_{i}^{-} \ell_{i}^{-}\right)+\ell_{j}^{+}+H_{T}
$$

The $4 \ell+E_{T}$ signal receives contributions from the pair-production of both chiral states of the doubly charged Higgsino. We add up their individual contributions to obtain the total number of events. This yields a rather clean and robust $4 \ell+$ missing $p_{T}$ signal at the LHC with highly suppressed SM background.

After required kinematic cuts [7], the total cross section for the $2 \mu^{-}+2 e^{+}+Z_{T}$ signal for SPA point is $7.71 \mathrm{fb}(\mathbf{S 2})$ and $7.02 \mathrm{fb}(\mathbf{S 3})$ while for SPB it is $2.43 \mathrm{fb}(\mathbf{S 2})$ and $2.66 \mathrm{fb}(\mathbf{S 3})$. In the case, where the doubly charged Higgsino is produced in association with a chargino, we have used the $\mathbf{S P C}$ point, and the corresponding cross sections for $2 \mu^{-}+e^{+}+E_{T}$ are $2.24 \mathrm{fb}(\mathbf{S 2})$ and 2.03 $\mathrm{fb}(\mathbf{S 3})$, respectively. As in pair-production, the $\widetilde{\Delta}^{--}$decays again into a pair of SSSF leptons and an LSP, while the chargino decays with almost $100 \%$ branching ratio to a neutrino and slepton for SPC. In Fig. 2 we show the binwise invariant mass distribution of lepton pairs for both SPA, SPB points and both cases of $\mathbf{S 2}$ and $\mathbf{S 3}$. These plots show differences in invariant mass distributions between the SSSF and opposite-sign-different-flavor (OSDF) lepton pairs. The SSSF lepton pairs exhibit a sharp kinematic edge in $M_{\ell \ell}$ distribution according to

$$
M_{\ell^{ \pm} \ell^{ \pm}}^{\max }=\sqrt{M_{\widetilde{\Delta}}^{2}+M_{\tilde{\chi}_{1}^{0}}^{2}-2 M_{\widetilde{\Delta}} E_{\widetilde{\chi}_{1}^{0}}},
$$

where $E_{\widetilde{\chi}_{1}^{0}}$ is the energy of the LSP, whereas the OSDF lepton pairs do not. The edge in the SSSF dilepton invariant mass distribution yields a clear hint of a $\Delta L=2$ interaction and a doubly charged field in the underlying model of 'new physics'. A very similar feature is expected for SPC point and the $3 \ell+E_{T}$ signal. The invariant mass distribution and also the distribution in $\Delta R$ of a pair 


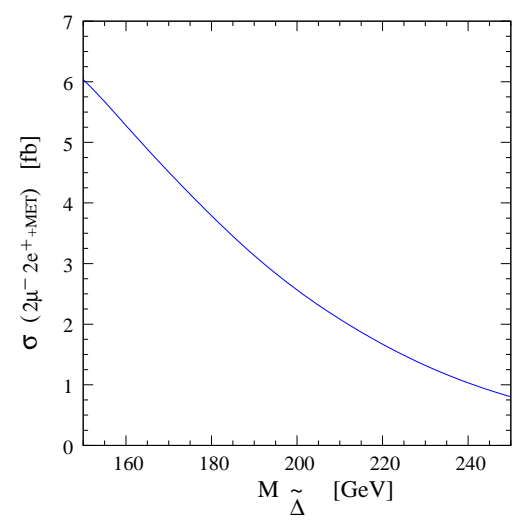

Figure 3: 4-lepton+MET production cross section at Tevatron.

SSSF and OSDF leptons, prove to be a very effective discriminant when compared with similar signals coming from other new physics scenarios [7].

\section{Discussion on Doubly Charged Higgsinos at Tevatron}

One may wonder if it is possible to detect the doubly charged Higgsinos already at the Tevatron, given the present mass limits. This is investigated in [8]. We assume that the sleptons and charginos are heavier than the doubly charged Higgsinos. After proper selection cuts [8], we show in Fig. 3 the cross section for the production of $2 \mu^{-} 2 e^{+}+E_{T}$. If we take also the charge conjugate state, the cross section doubles. The SM background for $2 \mu 2 e$ is small, $\sim 0.3 \mathrm{fb}$. We conclude that this signal should be visible for doubly charged Higgsino with mass up to around $200 \mathrm{GeV}$, see [8].

\section{References}

[1] Z. Chacko and R.N. Mohapatra, Phys. Rev. D 58, 015003 (1998); B. Dutta and R.N. Mohapatra, Phys. Rev. D 59, 015018 (1999).

[2] D.A. Demir, M. Frank and I. Turan, Phys. Rev. D 73, 115001 (2006).

[3] R.N. Mohapatra and A. Rasin, Phys. Rev. Lett. 76, 3490 (1996); R.N. Mohapatra and A. Rasin, Phys. Rev. D 54, 5835 (1996); R. Kuchimanchi, Phys. Rev. Lett. 76, 3486 (1996).

[4] M. Cvetic and J. Pati, Phys. Lett. B 135, 57 (1984); R.N. Mohapatra and A. Rasin, Phys. Rev. D 54, 5835 (1996); R. Kuchimanchi, Phys. Rev. Lett. 76, 3486 (1996); R.N. Mohapatra, A. Rašin and G. Senjanović, Phys. Rev. Lett. 79, 4744 (1997); C.S. Aulakh, K. Benakli and G. Senjanovic, Phys. Rev. Lett. 79, 2188 (1997); C.S. Aulakh, A. Melfo and G. Senjanovic, Phys. Rev. D 57, 4174 (1998).

[5] R.M. Francis, M. Frank and C.S. Kalman, Phys. Rev. D 43, 2369 (1991); K. Huitu, J. Maalampi and M. Raidal, Phys. Lett. B 328, 60 (1994); K. Huitu, J. Maalampi and M. Raidal, Nucl. Phys. B420, 449 (1994); K. Huitu and J. Maalampi, Phys. Lett. B 344, 217 (1995).

[6] M. Frank, K. Huitu and S.K. Rai, Phys. Rev. D 77, 015006 (2008).

[7] D.A. Demir, M. Frank, K. Huitu, S.K. Rai and I. Turan, Phys. Rev. D 78, 035013 (2008).

[8] D.A. Demir, M. Frank, D.K. Ghosh, K. Huitu, S.K. Rai, I. Turan, in preparation. 